Article

\title{
Antioxidant Effects of the Prenylated Flavonoid, Xanthohumol, on Corneal Epithelial Cells in Experimental Dry Eye Disease
}

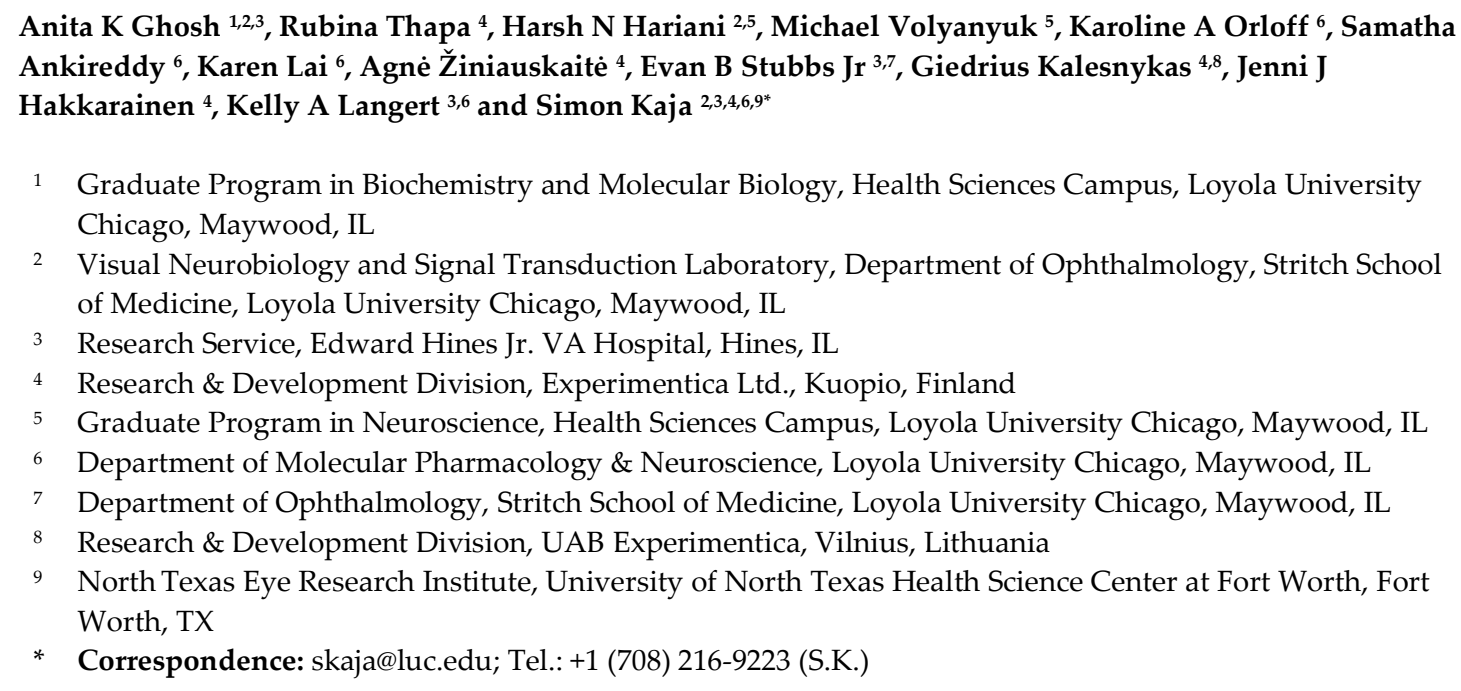

\begin{abstract}
Elevated levels of oxidative stress in the corneal epithelium contribute to the progression of dry eye disease pathology. Previous studies have shown that antioxidant therapeutic intervention is a promising avenue to reduce disease burden and slow disease progression. In this study, we evaluated the pharmacological efficacy of Xanthohumol in preclinical models for dry eye disease. Xanthohumol is a naturally occurring prenylated chalconoid that promotes the transcription of phase II antioxidant enzymes. Xanthohumol exerted a dose-response in preventing tertbutylhydroxide-induced loss of cell viability in human corneal epithelial (HCE-T) cells and resulted in a significant increase in expression of nuclear factor erythroid 2-related factor 2 (Nrf2), the master regulator of the endogenous antioxidant system. Xanthohumol-encapsulating poly(lactic-coglycolic acid) nanoparticles (PLGA NP) were cytoprotective against oxidative stress in vitro, and significantly reduced corneal fluorescein staining in the mouse desiccating stress/ scopolamine model for dry eye disease in vivo by reducing oxidative stress-associated DNA damage in corneal epithelial cells. PLGA NP represent a safe and efficacious drug delivery vehicle for hydrophobic small molecules to the ocular surface. Optimization of NP-based antioxidant formulations with the goal to minimize instillation frequency may represent future therapeutic options for dry eye disease and related ocular surface disease.
\end{abstract}

Keywords: ocular surface disease; dry eye disease; antioxidant; Xanthohumol; drug delivery; drug formulation; PLGA; nanoparticles

\section{Introduction}

Dry eye disease presents in various clinical manifestations that pose a substantial burden on the affected individual and society as a whole. Existing pharmacologic management for dry eye disease targets $\mathrm{T}$ cell-mediated inflammatory pathways and is associated with limited efficacy and adverse effects in up to $25 \%$ of patients [1-3], highlighting an urgent unmet clinical need for novel efficacious and well-tolerated therapeutics. 
Previous studies have implicated the generation of Reactive Oxygen Species (ROS) and the ensuing elevated levels of cellular oxidative stress as a key contributor to the pathophysiology of dry eye disease (reviewed in [4]). Specifically, elevated levels of oxidative stress have been identified in patients with dry eye disease $[5,6]$, while hyperosmolar conditions cause oxidative stress in cultured corneal epithelial cells [7]. We have recently shown significant oxidative DNA damage in the corneal epithelium of mice exposed to dry eye inducing conditions of desiccating environment with scopolamine [8]. Similarly, lacrimal gland dysfunction as a result of mitochondrial oxidative stress produces an ocular phenotype reminiscent of dry eye disease in mice [9, 10].

Notably, a mitochondrially-targeted antioxidant, SkQ1 (Visomitin) exerts anti-inflammatory effects in human conjunctival epithelial cells in vitro [11], and has shown therapeutic benefit in US Phase 2 clinical trials following approval in Russia in 2011 [12], providing proof-of-concept evidence supporting the development of therapeutic approaches using antioxidants to treat dry eye disease.

Major challenges associated with dry eye disease management are poor patient satisfaction and compliance with dosing regimens [13]. Therefore, one important drug development consideration for topical ophthalmic formulations is to enhance ocular surface retention times that minimize the number of instillations.

In this study, we evaluated the anti-oxidative and anti-inflammatory properties of Xanthohumol in preclinical models for dry eye disease. Xanthohumol is a naturally occurring prenylated chalconoid that is abundantly present in Humulus lupulus, the hops plant. Xanthohumol promotes the transcription of phase II antioxidant enzymes [14], by stimulating the dissociation of Kelch-like ECH-associated protein 1 (Keap1) from Nuclear factor erythroid 2-related factor 2 (Nrf2), the master regulator of the endogenous antioxidant response. Keap1 is the main negative regulator of Nrf2 targeting it for ubiquitylation and degradation. The dissociation of Keap1 from Nrf2 results in nuclear translocation of Nrf2 and subsequent activation of gene expression driven by the antioxidant response element. In addition, Xanthohumol exhibits direct ROS scavenging activity due to its chalconoid structure [15].

Xanthohumol was selected based on the rationale that exploiting its dual mechanism of boosting the endogenous antioxidant response by relieving Keap1 suppression of Nrf2 translocation and direct ROS scavenging may be advantageous over antioxidants with only direct ROS scavenging activity.

The objectives of this study were to determine the cytoprotective effects of Xanthohumol in human corneal epithelial cells in vitro, and in the mouse desiccating stress/ scopolamine model for dry eye disease in vivo, using both non-formulated and poly(lactic-co-glycolic acid) nanoparticle (PLGA NP)-encapsulating Xanthohumol.

\section{Results}

\subsection{Xanthohumol exerts cytoprotective effects against chemically-induced oxidative stress in HCE-T cells}

In order to determine the cytotoxicity of Xanthohumol, human corneal epithelial (HCE-T) cells were exposed to a concentration range of Xanthohumol $(10 \mathrm{nM}-100 \mu \mathrm{M})$ and incubated for $48 \mathrm{~h}$. Dimethyl sulfoxide vehicle was kept constant at $0.1 \%$ volume/volume for all Xanthohumol concentrations. Cell survival and proliferation were assessed by 3-(4,5-dimethylthiazol-2-yl)-2,5diphenyltetrazolium bromide (MTT) uptake and lactate dehydrogenase (LDH) release assays. Xanthohumol concentrations up to $10 \mu \mathrm{M}$ had no effect on MTT absorbance (Figure 1A) or LDH release (Figure 1B). Higher concentrations of Xanthohumol exerted dose-dependent cytotoxicity, resulting in almost complete loss of cell viability at $100 \mu \mathrm{M}$ (Figure 1). 
A

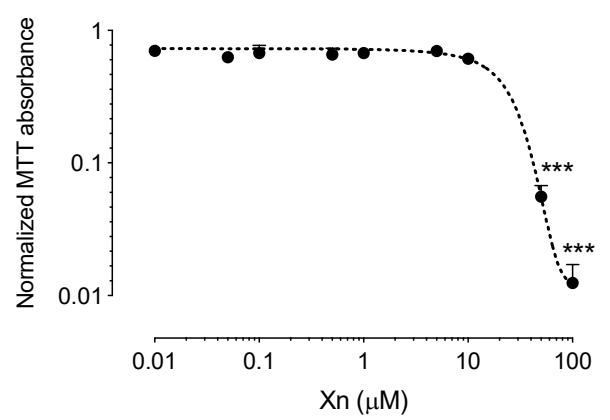

B

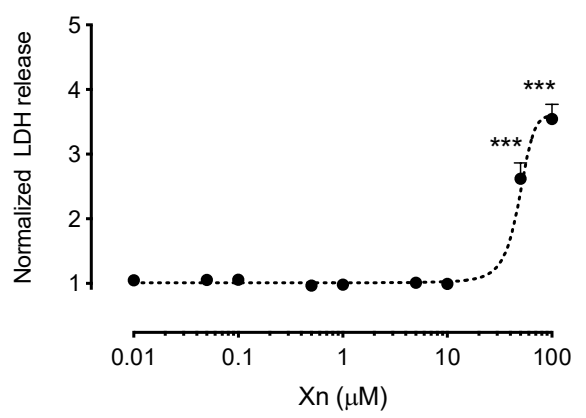

Figure 1. Cytotoxicty of Xanthohumol in HCE-T cells. (a) Xanthohumol resulted in dose-dependent cytotoxicity in HCE-T cells as shown by MTT assay. Concentrations up to $10 \mu \mathrm{M}$ did not have a statistically significant effect on cell proliferation and survival. Concentrations of $50 \mu \mathrm{M}$ and $100 \mu \mathrm{M}$ resulted in a reduction of MTT absorbance by $94.4 \pm 1.2 \%$ and $98.8 \pm 0.5 \%$, respectively $(\mathrm{n}=3)$; $(\mathbf{b})$ Similarly, Xanthohumol concentrations greater than $10 \mu \mathrm{M}$ resulted in a statistically significant increase in LDH release (2.6 \pm 0.2 -fold at $50 \mu \mathrm{M}$ and 3.5 \pm 0.2 -fold at $100 \mu \mathrm{M} ; \mathrm{n}=3)$. Data are shown as mean \pm SEM. ${ }^{* * *} P<0.001$. $\mathrm{Xn}=$ Xanthohumol.

Based on the results from cytotoxicity assays, we selected four sublethal concentrations of Xanthohumol $(0.1 \mu \mathrm{M}, 0.5 \mu \mathrm{M}, 1 \mu \mathrm{M}$ and $5 \mu \mathrm{M})$ to determine the cytoprotective and antioxidant effects against exogenously-applied tert-butyl hydroperoxide $(t \mathrm{BHP})$-induced oxidative stress. HCE$\mathrm{T}$ cells were exposed to Xanthohumol for $20 \mathrm{~h}$, and subsequently exposed to a concentration range of tBHP $(5-500 \mu \mathrm{M})$ for $6 \mathrm{~h}$ prior to performing MTT and LDH assays (Figure 2). For these studies, dimethyl sulfoxide vehicle was used at a concentration of $0.005 \%$ volume/volume.

A

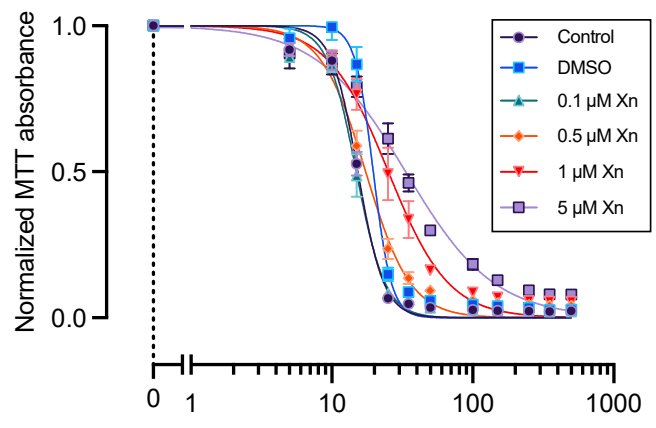

C

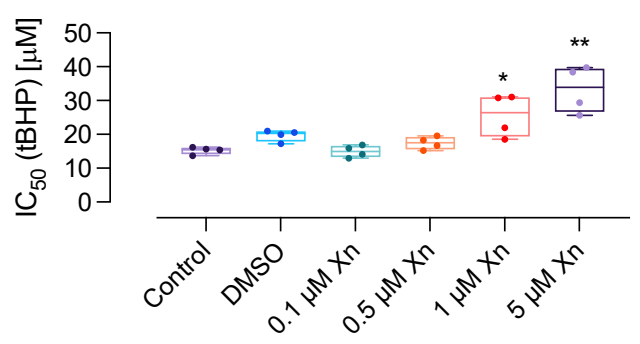

B

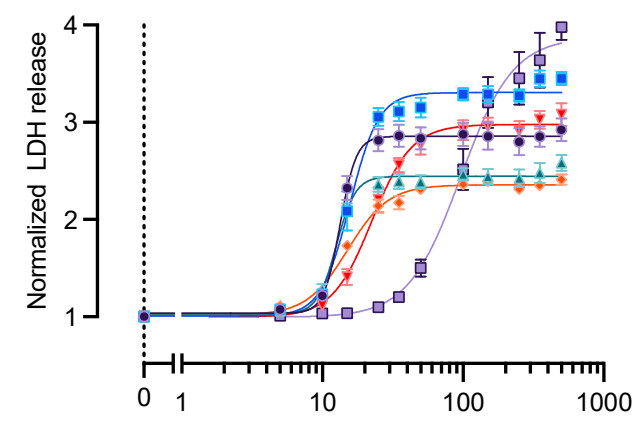

D

$[\mathrm{tBHP}](\mu \mathrm{M})$

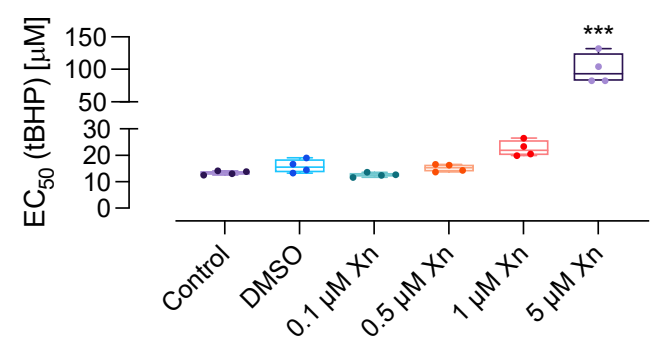

Figure 2. Xanthohumol exerts dose-dependent cytoprotective effects against $t \mathrm{BHP}$-induced oxidative stress in HCE-T cells. (a) Xanthohumol $(0.1-5 \mu \mathrm{M})$ caused a right-shift of dose-response curves for tBHP in the MTT assay, suggestive of cytoprotection. Data were fitted using a four-parameter dose response curve. (b) Similarly, Xanthohumol $(0.1-5 \mu \mathrm{M})$ resulted in a right-shift of the LDH response. 
(c) Quantification of the $\mathrm{EC}_{50}$ values from the MTT assay revealed an approximately 2.2-fold increase in the presence of $5 \mu \mathrm{M}$ Xanthohumol $(15.2 \pm 0.5 \mu \mathrm{M}$ vs. $33.3 \pm 3.4 \mu \mathrm{M}, P<0.01, \mathrm{n}=4)$. (d) In the LDH assay, Xanthohumol $(5 \mu \mathrm{M})$ increased the EC50 for $t \mathrm{BHP}$ from $13.4 \pm 0.4 \mu \mathrm{M}$ to $100.0 \pm 11.7 \mu \mathrm{M}(P<$ $0.001 ; \mathrm{n}=4)$. Data are shown as mean \pm SEM. ${ }^{*} P<0.05,{ }^{* *} P<0.01,{ }^{* *} P<0.001 . \mathrm{Xn}=$ Xanthohumol.

Xanthohumol resulted in a dose-dependent protection against oxidative stress, as evident by a right-shift in the IC 50 curves for $t \mathrm{BHP}$ in the MTT assay (Figure 2A). Similarly, Xanthohumol caused a right-shift in the $\mathrm{EC}_{50}$ curves for $t \mathrm{BHP}$ in the LDH assay (Figure $2 \mathrm{~B}$ ). Specifically, the $\mathrm{IC}_{50}$ for $t \mathrm{BHP}$ in the MTT assay was $15.2 \pm 0.5 \mu \mathrm{M}$ in the control condition. $1 \mu \mathrm{M}$ and $5 \mu \mathrm{M}$ Xanthohumol resulted in a statistically significant increase in the $\mathrm{EC}_{50}$ values for $t \mathrm{BHP}$ to $25.6 \pm 3.2 \mu \mathrm{M}(P<0.05, \mathrm{n}=4)$ and $33.3 \pm 3.4 \mu \mathrm{M}(P<0.01, \mathrm{n}=4$; Figure $2 \mathrm{C})$, respectively.

Similarly, Xanthohumol $(5 \mu \mathrm{M})$ increased the $\mathrm{EC}_{50}$ value for $t \mathrm{BHP}$ in the LDH assay from $13.4 \pm$ $0.4 \mu \mathrm{M}$ to $100.0 \pm 11.7 \mu \mathrm{M}(P<0.001 ; \mathrm{n}=4$; Figure $2 \mathrm{D})$.

\subsection{Xanthohumol elicits significant increase in Nrf2 protein levels in human corneal epithelial cells}

Xanthohumol is a well-known activator of the endogenous antioxidant system that acts by stimulating the dissociation of Keap1 from Nrf2. In order to demonstrate the ability of Xanthohumol to elicit this effect in corneal epithelial cells, we performed a time course analysis of Nrf2 protein levels after exposure to Xanthohumol in HCE-T cells.

Nrf2 protein levels peaked after $6 \mathrm{~h}$ of Xanthohumol $(5 \mu \mathrm{M})$ and were $5.0 \pm 1.7$-fold higher than in vehicle-treated cells $(1.0 \pm 0.2 ; n=3, P<0.01$; Figure 3$)$.

Together with results from the cell viability assays presented in Figure 2, our data suggest that Xanthohumol can exert antioxidant effects in human corneal epithelial cells.

A

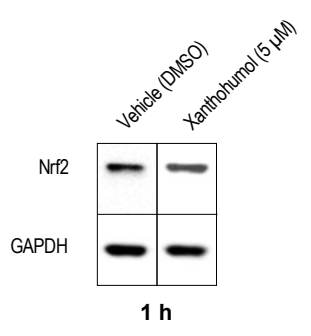

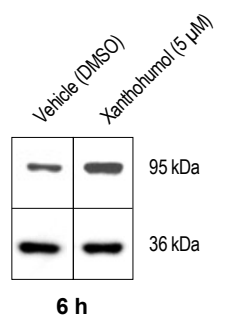

B

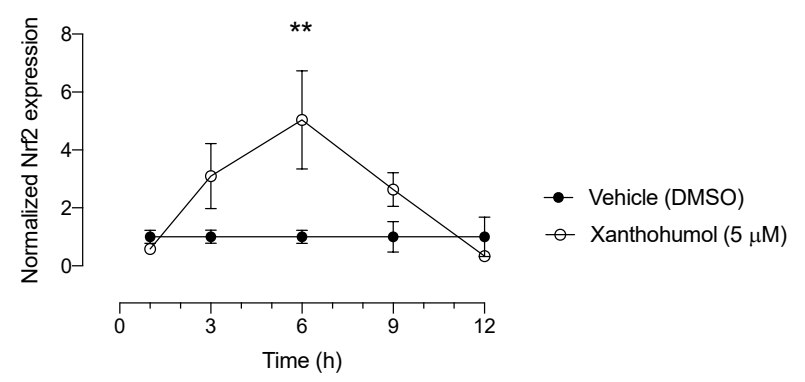

Figure 3. Xanthohumol increases protein levels of Nrf2 in human corneal epithelial cells. (a) Representative examples of Nrf2 immunoblots from Xanthohumol- versus vehicle-treated cell lysates are shown. GAPDH was used as endogenous control. (b) Quantification of immunoblots revealed a statistically significant 5-fold increase of Nrf2 in Xanthohumol-treated cells compared with vehicle after $6 \mathrm{~h}$ incubation. Data are shown as mean \pm SEM from three separate experiments. ${ }^{* *} P<0.01$.

\subsection{Xanthohumol-encapsulating PLGA NP are cytoprotective against oxidative stress in HCE-T cells}

We next generated Xanthohumol-encapsulating PLGA NP using an 85:15 ratio of poly-lactic and poly-glycolic acid, based on previously established release parameters [16]. Nanoparticle formulations were resuspended in saline and their properties analyzed by Dynamic Light Scattering using a ZetaSizer (Malvern Pananalytical Inc., Westborough, MA, USA). Encapsulation efficiency of Xanthohumol was $68.8 \%$ (data not shown).

Empty and Xanthohumol-encapsulating PLGA NP were similar in size and size distribution averaging $200 \mathrm{~nm}$ (Figure 4; Table 1). Similarly, the polydispersity index was below 0.05 for both PLGA NP formulations, suggesting a unimodal size distribution and absence of aggregation (Table 1). The surface charge of PLGA NPs was negative, in line with previous observations [16] (Table 1). 


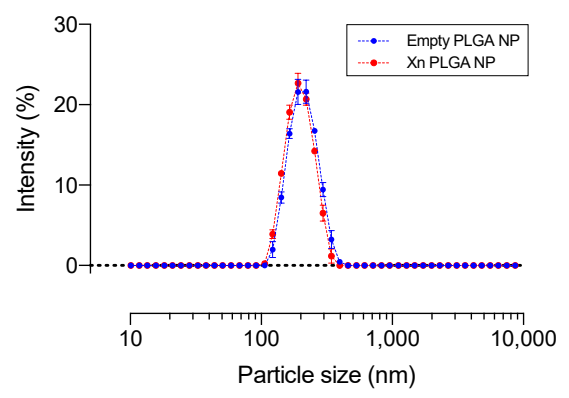

Figure 4. Size distribution of PLGA NP. Empty and Xanthohumol-encapsulating PLGA NP have similar sizes and unimodal size distribution. Data are mean \pm SEM from three different PLGA NP preparations, each tested in triplicate. $\mathrm{Xn}=$ Xanthohumol.

To assess the cytotoxicity of PLGA NP and release of Xanthohumol, we performed cell viability assays in HCE-T cells analogous to the experiments described above. HCE-T cells were seeded in 96 well plates and incubated with increasing amounts of empty and Xanthohumol-encapsulating PLGA $\mathrm{NP}$ for $48 \mathrm{~h}$. The concentration of Xanthohumol represents the total amount of Xanthohumol present in the NP applied to the cells. In the control condition, cells were exposed to an equivalent amount (milligrams) of empty PLGA NP.

Table 1. Properties of PLGA NP formulations.

\begin{tabular}{ccc}
\hline Parameter & Empty PLGA NP & Xn PLGA NP \\
\hline Size $(\mathrm{nm})$ & $201.9 \pm 0.1$ & $191.0 \pm 0.8$ \\
Polydispersity Index $(\mathrm{PDI})$ & $0.045 \pm 0.009$ & $0.029 \pm 0.007$ \\
Zeta $(\zeta)$ potential $(\mathrm{mV})$ & $-21.6 \pm 0.3$ & $-24.8 \pm 0.2$ \\
\hline
\end{tabular}

Increasing concentrations of Xanthohumol-encapsulating PLGA NP exerted a dose-dependent toxicity as evident by a decrease in MTT absorbance $(n=3-5, P<0.001$; Figure 5A) and a concomitant increase in LDH release ( $\mathrm{n}=3-5 ; P<0.001$; Figure $5 \mathrm{~B})$. In contrast, increasing amounts (matching the NP amount of each Xn NP dose) of empty PLGA NP did not exert any cytotoxicity (Figure 5A, B). Differences were statistically evaluated by Two-Way ANOVA with Šídák's multiple comparisons test, indicating that concentration of $10 \mu \mathrm{M}$ or higher resulted in statistically significant cytotoxicity in HCE-T cells.

A

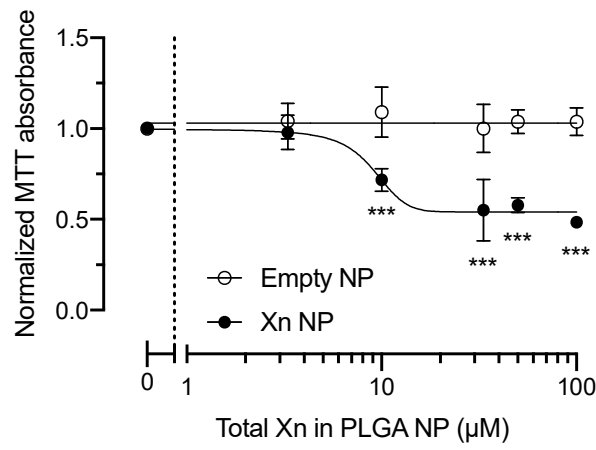

B

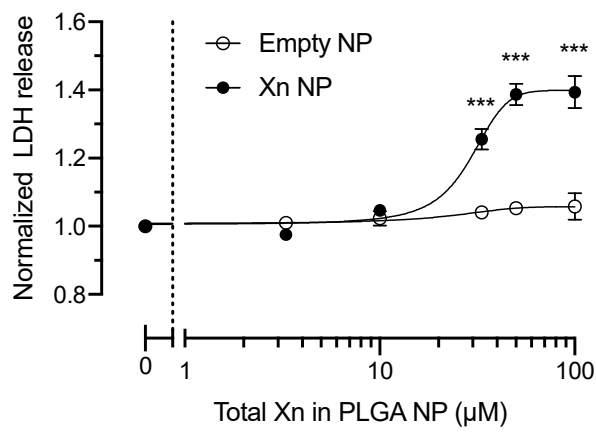

Figure 5: Cytotoxicity of Xanthohumol-encapsulating PLGA NP in HCE-T cells. (a) Xanthohumolencapsulating PLGA NP resulted in dose-dependent cytotoxicity in HCE-T cells as shown by MTT assay. Concentrations $\geq 10 \mu \mathrm{M}$ exerted a statistically significant effect on cell proliferation and survival after $48 \mathrm{~h}$ incubation when compared to matching amount of empty PLGA NP ( $\mathrm{n}=3-5, P<$ 0.001); (b) Similarly, Xanthohumol concentrations of $>10 \mu \mathrm{M}$ resulted in a statistically significant increase of LDH release ( $\mathrm{n}=3-5, P<0.001)$. Data were analyzed by Two-Way ANOVA with Šídák's 
multiple comparisons test and are shown as mean \pm SEM. ${ }^{* *} P<0.001 . \mathrm{Xn}=$ Xanthohumol, NP $=$ nanoparticle, PLGA = poly(lactic-co-glycolic acid)

Next, we tested the ability of Xanthohumol-encapsulating NP to protect HCE-T cells from exogenously-applied oxidative stress. We incubated HCE-T cells with either empty or Xanthohumolencapsulating $(5 \mu \mathrm{M})$ PLGA NP for $20 \mathrm{~h}$, prior to exposing HCE-T cells to a dose-range of tBHP (25 $125 \mu \mathrm{M}$ ) for $5 \mathrm{~h}$. Xanthohumol-encapsulating PLGA NP caused a statistically significant shift in the dose-response to $t \mathrm{BHP}(\mathrm{n}=3, P<0.01$; Figure $6 \mathrm{~A})$, with $\mathrm{IC}_{50}$ values for $t \mathrm{BHP}$ increasing from $16.6 \mu \mathrm{M}$ (interquartile range: $14.1 \mu \mathrm{M}-18.9 \mu \mathrm{M}$ ) to $21.2 \mu \mathrm{M}$ (interquartile range: $17.9 \mu \mathrm{M}$ to $24.1 \mu \mathrm{M}$ ). Similarly, $\mathrm{EC}_{50}$ for $\mathrm{tBHP}$ values derived from the LDH release assay increased significantly from 17.9 $\mu \mathrm{M}$ to $22.4 \mu \mathrm{M}(\mathrm{n}=3, P<0.01$; Figure $6 \mathrm{~B})$.
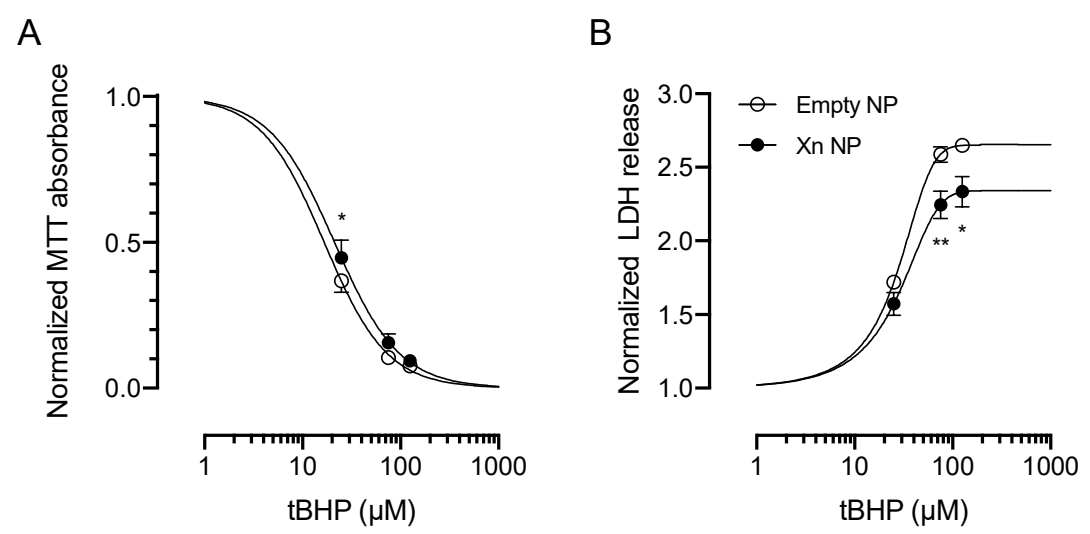

Figure 6. Xanthohumol-encapsulating PLGA NP showed cytoprotection against $t$ BHP-induced oxidative stress in HCE-T cells. (a) Xanthohumol-encapsulating PLGA NP (5 $\mu \mathrm{M})$ caused a right-shift of the dose-response curves for $\mathrm{tBHP}$ in the MTT assay, shifting the EC50 value by $4.6 \mu \mathrm{M}(\mathrm{n}=3, P<$ 0.05). (b) Similarly, Xanthohumol-encapsulating PLGA NP $(5 \mu \mathrm{M})$ resulted in a right-shift of the LDH response, increasing the $\mathrm{IC}_{50}$ value by $4.5 \mu \mathrm{M}(\mathrm{n}=3, P<0.01)$. Data are shown as mean \pm SEM and were fitted using a four-parameter dose response curve. Data were analyzed by Two-Way ANOVA followed by Šídák's multiple comparisons test. ${ }^{*} P<0.05,{ }^{*} P<0.01$. Xn NP $=$ Xanthohumolencapsulating PLGA NP.

Based on these data, we have identified a safe dose of Xanthohumol-encapsulating PLGA NP in HCE-T cells and confirm that Xanthohumol delivered via PLGA NP can exert antioxidative effects in human corneal epithelial cells. In the next set of experiments, we tested the efficacy of Xanthohumolencapsulating PLGA NP in a preclinical dry eye disease model.

\subsection{Xanthohumol-encapsulating PLGA NP are cytoprotective against oxidative stress in HCE-T cells}

We used the mouse desiccating stress/ scopolamine model to test the efficacy of Xanthohumolencapsulating PLGA NP. Mice were exposed to SiccaSystem ${ }^{\circledR}$ cages for a period of 15 days without intervention. Subsequently, mice were treated twice daily ( $8 \mathrm{am}$ and $6 \mathrm{pm})$ by topical instillation of either empty PLGA NP, Xanthohumol-encapsulating PLGA NP or cyclosporine. In this study, we did not include a separate vehicle control group, as we have previously determined that empty PLGA NP do not exert any cytoprotective effects compared with $0.9 \%$ saline solution (data not shown).

First, we quantified tear volumes, at baseline, before start of topical treatments on day 15 and at the end of the study on day 26. We observed a statistically significant reduction of tear volumes on Day 15 suggestive of successful induction of dry eye disease pathology (from $4.7 \pm 0.3 \mathrm{~mm}$ to $2.0 \pm 0.1$ $\mathrm{mm}, \mathrm{n}=60$ eyes, $P<0.001)$. Two Way ANOVA analysis revealed a statistically significant effect of time $(P<0.001)$, but not treatment $(P=0.29)$, and tear volumes showed a similar statistically significant increase of tear volumes from day 15 to day $26(\mathrm{P}<0.05$ for all treatment groups using Tukey's multiple comparisons test; Figure 7A). Effect sizes for each treatment, determined by 
calculating the difference between tear volume and day 15 and day 26, did also not differ between treatment groups (Kruskal-Wallis ANOVA, $P=0.86$; Figure 7B).

A

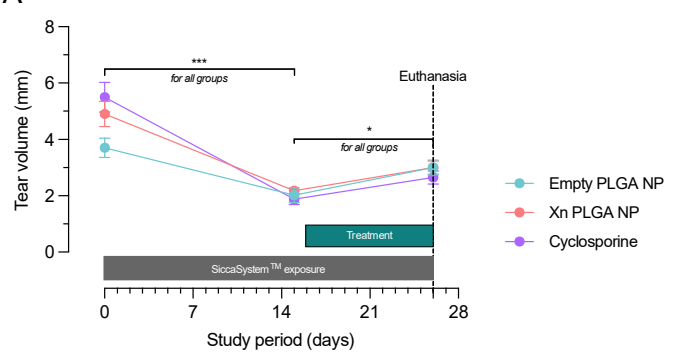

B

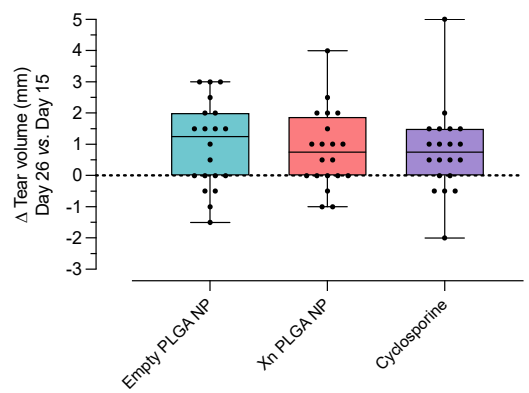

Figure 7. Xanthohumol PLGA NP do not affect tear volumes. (a) Tear volumes decreased significantly as a result of the exposure to the desiccating stress/scopolamine environment $(\mathrm{n}=20, P<0.001$ for all groups); all treatments significantly increased tear volumes ( $\mathrm{n}=20, P \leq 0.05$ for all groups), as determined by Two-Way ANOVA. Data are shown as mean \pm SEM $(\mathbf{b})$ Comparison of effect sizes determined by calculating the difference in tear volume between day 25 and day 16 did not show any statistically significant differences ( $\mathrm{n}=20, P=0.86$, Kruskal-Wallis ANOVA). Data are shown as box and whisker plot, indicating the median (line), with the box extending from the $25^{\text {th }}$ to $75^{\text {th }}$ percentiles. Whiskers represent the range, while filled circles are individual data points from a single eye. $\mathrm{Xn}=$ Xanthohumol, NP = nanoparticle, PLGA = poly (lactic-co-glycolic acid)

In order to determine possible effects on corneal damage, we performed corneal fluorescein staining, again before start of topical treatments on day 15 and at the end of the study on day 26 (Figure 8A). Corneal fluorescein staining was quantified by determining the fluorescence intensity of fluorescein on the cornea. Empty PLGA NP did not significantly affect corneal fluorescein staining $(P=0.21 ;$ Figure 8B). In contrast, Xanthohumol-encapsulating PLGA NP $(P<0.05)$ and cyclosporine $(P<0.01)$ treatment resulted in a statistically significant reduction of corneal fluorescein staining.

A Empty PLGA

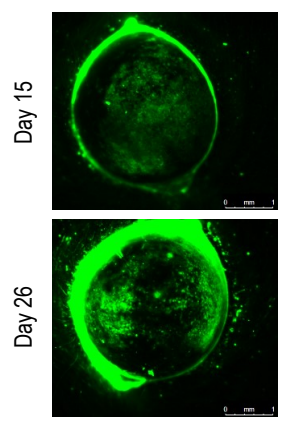

Xn PLGA

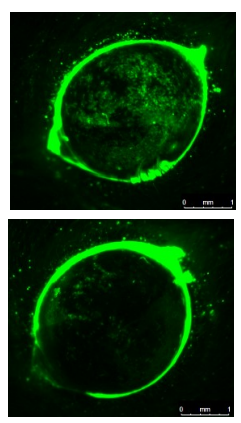

Cyclosporine

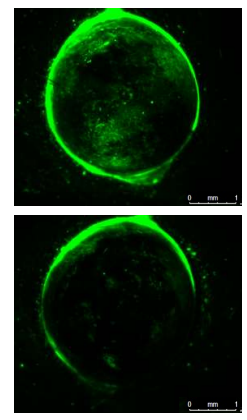

$\mathrm{B}$

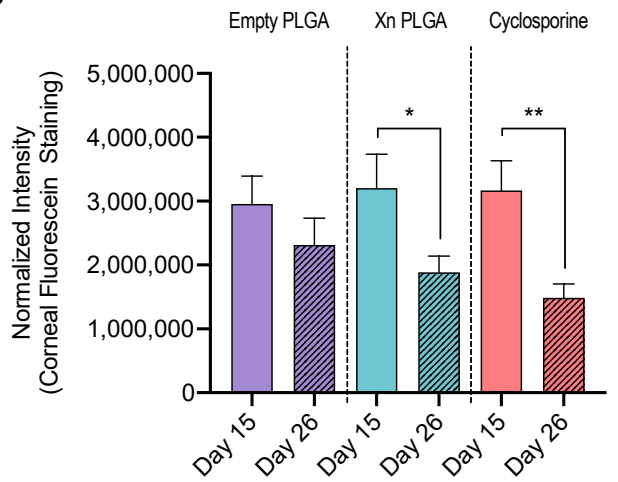

Figure 8: Xanthohumol-encapsulating PLGA NP reduce corneal fluorescein staining in the mouse desiccating stress/ scopolamine model for dry eye disease. (a) Representative examples of corneal fluorescein staining from day 15 and day 26 . Scale bar: $1 \mathrm{~mm}$. (b) Quantification revealed a statistically significant reduction of corneal fluorescein staining by Xanthohumol-encapsulating PLGA NP and cyclosporine, while empty PLGA NP had no significant effect on fluorescein staining $(n=18-20$ eyes per group), as determined by Mann-Whitney test. ${ }^{*} P<0.05$, ${ }^{* *} P<0.01$. Data are shown as mean \pm SEM. $\mathrm{Xn}=$ Xanthohumol, PLGA $=$ poly (lactic-co-glycolic acid $)$

We have previously shown that the desiccating stress/ scopolamine model results in a significant amount of oxidative DNA damage that can be prevented by antioxidant treatment [17]. In order to 
determine the efficacy of Xanthohumol-encapsulating PLGA NP, we stained corneal sections for 8hydroxy-2' deoxyguanosine (8-OHdG) and quantified immunoreactivity in corneal epithelial cells. Empty PLGA NP-treated eyes showed significant nuclear 8-OHdG immunoreactivity; in contrast, Xanthohumol-encapsulating PLGA NP showed a visible reduction in 8-OHdG intensity (Fig. 9A). Quantification revealed a statistically significant reduction in 8-OHdG staining by $49.3 \pm 7.3 \%(\mathrm{n}=9$ - 10 per group; $P<0.01$; Fig. 9B).

A

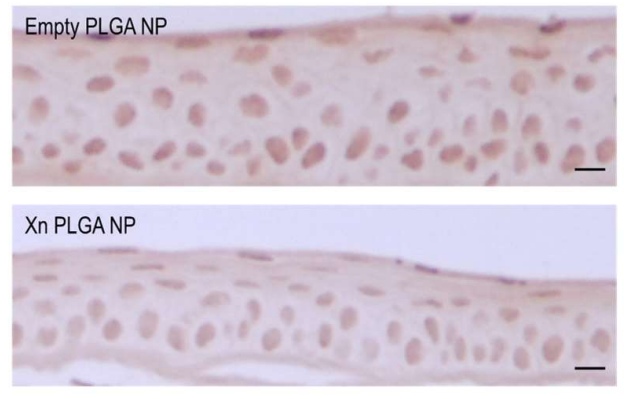

B

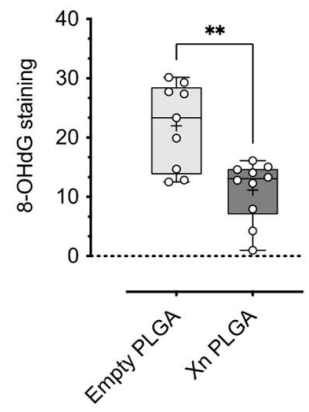

Figure 9: Xanthohumol-encapsulating PLGA NP reduce 8-OHdG immunoreactivity in corneal epithelial cells. (a) Representative examples of 8-OHdG immunoreactivity in corneal epithelial cells in empty and Xanthohumol-encapsulating PLGA NP-treated eyes. (b) Quantification of nuclear intensity revealed a statistically significant reduction of $8-\mathrm{OHdG}$ staining by Xanthohumolencapsulating PLGA NP compared to empty PLGA NP. Data are shown as box and whisker plot, indicating the median (line), with the box extending from the $25^{\text {th }}$ to $75^{\text {th }}$ percentiles. Whiskers represent the range, while filled circles are individual data points from a single eye $(n=9-10$ per group); the mean is indicated by a plus $(+)$ sign. ${ }^{* *} P<0.01$. Scale bar: $10 \mu \mathrm{M}$.

This marked reduction in oxidative stress-associated DNA damage in corneal epithelial cells was not associated with marked changes in the histopathological properties of the cornea (Table 2). Specifically, epithelial and stromal thickness were not significantly affected by Xanthohumolencapsulating PLGA NP treatment.

Table 2. Histopathological properties of corneal tissue.

\begin{tabular}{cccc}
\hline Parameter & Empty PLGA NP & Xn PLGA NP & Statistics \\
\hline Thickness, corneal epithelium $(\mu \mathrm{m})^{*}$ & $35.5 \pm 3.3$ & $30.8 \pm 2.2$ & $\mathrm{n}=10, P=0.24$ \\
Thickness, corneal stroma $(\mu \mathrm{m})^{*}$ & $178.9 \pm 12.9$ & $159.4 \pm 7.8$ & $\mathrm{n}=10, P=0.21$ \\
Number of epithelial cell layers ${ }^{* *}$ & $5(4 ; 5.5)$ & $4(4 ; 5)$ & $\mathrm{n}=10, P=0.24$ \\
\hline
\end{tabular}

$*$ Data are shown as mean $\pm \mathrm{SEM}$, or $* *$ median $\left(25^{\text {th }}\right.$ percentile; $75^{\text {th }}$ percentile $)$

\section{Discussion}

Our data provide strong in vitro and in vivo evidence that the natural compound, Xanthohumol, can exert cytoprotective and antioxidative effects in preclinical models for dry eye disease. Specifically, Xanthohumol and Xanthohumol-encapsulating PLGA NP were cytoprotective against oxidative stress injury in human corneal epithelial cells. Furthermore, Xanthohumol-encapsulating PLGA NP delivered topically reduced severity of corneal fluorescein staining and 8-OHdG labeling in the cornea, suggestive of reduced corneal damage and corneal oxidative DNA damage, respectively.

Previous studies have implicated increased cellular levels of oxidative stress in ocular surface disease. For example, lacrimal gland dysfunction can cause hyperosmolarity of the tear film [18], eliciting the generation of oxidative stress in human corneal epithelial cells [7]. Reactive Oxygen Species can activate nuclear factor- $\kappa \mathrm{B}(\mathrm{NF}-\kappa \mathrm{B})$ [19], which regulates both the endogenous antioxidant system, but also pro-inflammatory signaling through toll-like receptor 4 [20]. In our previous studies, 
we have shown that exposure to the desiccating stress/ scopolamine model for dry eye disease causes significant increases in oxidative stress-mediated corneal damage [17], extending previous reports of apoptosis and damage to the corneal epithelium [19]. Therefore, the desiccating stress/ scopolamine model for dry eye disease is a useful model to investigate the effects of antioxidants and antioxidant formulations on the ocular surface.

We used HCE-T cells as in vitro model to determine toxicity and efficacy of Xanthohumol and Xanthohumol-encapsulating PLGA NP (Figures 1 - 3 and 5 - 6). While HCE-T cells are widely used, especially as they form a stratified epithelium with barrier properties and a characteristic morphology [21]; however, HCE-T cells also display genomic abnormalities suggestive of some genetic drift [22], which must be considered when interpreting in vitro findings derived from this cell line. Our mouse model for dry eye disease is based on a well-established paradigm that employs lowhumidity air flow and concurrent scopolamine administration to induce dry eye disease in wild-type mice [23]. We have previously refined the model and its quantitative readouts used to assess dry eye disease severity for determining the efficacy of novel anti-dry eye disease therapeutics, including antioxidants [17]. The magnitude of changes, as well as the response of the positive control, ophthalmic cyclosporine (Restasis), were similar to those reported by us and others previously for this model [17, 23, 24].

Exposure to the desiccating stress environment with concomitant scopolamine administration resulted in a statistically significant reduction of tear volumes $(\sim 60 \%)$, showing successful induction of ocular surface disease (Figure 7A). In this study, all groups showed a statistically significant increase in tear volumes at the end of the 10-day treatment period, however, no statistically significant differences between vehicle, Xanthohumol and cyclosporine-treated eyes were observed (Figure 7B). This suggests that the increase is primarily caused by lubrication of the cornea and tissues of the ocular surface, rather than due to a direct pharmacological effect. Here it may be important to note that tear volume measurements from mice are notoriously challenging and are easily confounded by physiological and environmental factors.

To determine the pharmacological efficacy of Xanthohumol in vivo, we used a PLGA NP-based formulation. PLGA NP are well-tolerated, biodegradable and approved by The United States Food and Drug Administration. Release from PLGA NP occurs as NP degrade and is governed, in part, by the ratio of poly-lactic and poly-glycolic acid parameters [25]. For this first proof-of-concept study, we used a ratio of 85:15 (poly-lactic:poly-glycolic acid), based on predicted release properties for the NP published by us and others previously $[16,26]$.

One shortcoming of the current study is that PLGA (85:15) NP are negatively charged (Table 1). It is generally assumed that cationic NPs exhibit enhanced retention times on negatively charged ocular tissues, such as the cornea and the conjunctiva [25, 27]. Therefore, we opted to administer Xanthohumol-encapsulating PLGA NP twice daily, matching the instillation frequency of cyclosporine. A detailed quantitative analysis of retention times of Xanthohumol-encapsulating PLGA NP on the ocular surface is beyond the scope of this article, which to our knowledge provides the first preclinical proof-of-concept supporting the use of Xanthohumol for ocular surface disease. Future studies will address modifications to PLGA NP formulations to include co-polymers such as chitosan or Eudragit RL100. For example, the latter, a copolymer of ethyl acrylate, methyl methacrylate and a low content of methacrylic acid ester with quaternary ammonium groups, has been successfully used for encapsulating cyclosporine with enhanced properties for topical delivery [28]. Nonetheless, Xanthohumol-encapsulating PLGA NP showed similar efficacy when compared against $0.05 \%$ ophthalmic cyclosporine emulsion (Restasis; Figure 8 ), which is the current standard of care for patients with moderate to severe dry eye disease in the United States [2, 29].

Xanthohumol is generally considered to exert its cytoprotective effects through both stimulating the dissociation of Keap1 from Nrf2 and direct ROS scavenging activity [14, 15]. Typically, scavenging of ROS results in the diminishing activation of the phase II antioxidant system [30], reducing the endogenous antioxidant potential as cellular levels of oxidative stress fall. Given the potent activation of Nrf2 in HCE-T cells in the absence of oxidative stress (Figure 3), Xanthohumol may be particularly well-suited for encapsulation in PLGA NP. In a previous study, we quantified the efficacy of three-times daily administration of the potent superoxide dismutase mimetic, 
manganese(III) tetrakis(1-methyl-4- pyridyl) porphyrin (Mn-TM-2-PyP). Intriguingly, Xanthohumolencapsulating PLGA NP had a much larger effect on 8-OHdG labeling in the cornea, reducing density of immunolabel by $\sim 50 \%$ (Figure 9), compared with an $25 \%$ reduction elicited by Mn-TM-2-PyP [17]. Given that the antioxidant potential of Mn-TM-2-PyP is significantly greater than that of Xanthohumol ([17, 31]), this finding may suggest that Xanthohumol-encapsulating PLGA NP are not only able to be retained at the ocular surface for a prolonged period of time despite their negative surface charge, but also achieve sustained activation of the endogenous antioxidant system.

\section{Materials and Methods}

\subsection{Test articles, antibodies and chemicals}

Xanthohumol was purchased from Cayman Chemicals (Ann Arbor, MI, USA) and dissolved in dimethyl sulfoxide at a concentration of $100 \mu \mathrm{M}$ (Millipore Sigma, St. Louis, MO, USA) for in vitro experiments. Cyclosporine A for transporter assays was USP grade ( $\leq 99 \%$ purity) from Cayman Chemical Company (Ann Arbor, MI, USA). Ophthalmic cyclosporine emulsion was pharmaceutical grade, Restasis ${ }^{\circledast}(0.05 \%$ cyclosporine; Allergan Plc., Irvine, CA, USA).

The following antibodies were used for immunoblotting experiments: mouse anti-NFE2L2 (Nrf2; VMA00224; BioRad Laboratories Inc., Hercules, CA; 1:1,000 dilution). Glyceraldehyde-3phosphate dehydrogenase (GAPDH) was used as endogenous control (rabbit anti-GAPDH; sc-25778; Santa Cruz Biotechnology, Dallas, TX; 1:2000 dilution). Secondary antibodies were horseradish peroxidase-conjugated and obtained from GE Healthcare (Chicago, IL, USA).

Unless otherwise specified, analytical grade reagents were obtained from Millipore Sigma (St. Louis, MO, USA).

\subsection{Cell culture}

Human corneal epithelial cells (HCE-T; RIKEN BioResource Research Center, Tsukuba, Japan) were cultured as described by us previously [17, 31-33]. Cultures of passages 79 to 95 , were used for experiments.

\subsection{Cell viability assays}

To determine the cytoprotective effects of Xanthohumol against chemically-induced oxidative stress, we conducted 3-(4,5-dimethylthiazol-2-yl)-2,5-diphenyltetrazolium bromide (MTT) uptake and lactate dehydrogenase (LDH) release assays, essentially as previously described by us in detail [17]. In brief, supernatants (50 $\mu \mathrm{L})$ were collected and LDH assays performed. Cells were incubated with MTT dye for $1.5 \mathrm{~h}$ and subsequently lysed in dimethyl sulfoxide. Data were normalized to the baseline control condition and expressed as fold-change.

\subsection{Quantitative immunoblotting}

Immunoblotting on HCE-T cell lysates was performed as described by us previously [31].

\subsection{Generation and characterization of PLGA NP}

PLGA NPs were prepared using an oil-in-water single emulsion technique, essentially as described previously [16]. Briefly, 50 mg of PLGA (85:15; Durect Corp., Birmingham, AL) were dissolved in $1 \mathrm{ml}$ dichloromethane and slowly added to ice-cold polyvinyl alcohol (1\% w/v, $10 \mathrm{ml})$, while vigorously vortexing. The resultant suspension was emulsified by probe sonication and diluted with $100 \mathrm{ml}$ ice-cold PVA. The organic solvent was allowed to evaporate with constant stirring for 3 $\mathrm{h}$ at $23^{\circ} \mathrm{C}$ and the resulting PLGA nanoparticles were isolated by centrifugation $(25,000 \times \mathrm{g}$ for $20 \mathrm{~min}$ at $\left.4{ }^{\circ} \mathrm{C}\right)$ and washed three times with deionized water. PLGA NP were resuspended in sucrose (10 $\mathrm{ml}$ of $5 \mathrm{mg} / \mathrm{ml}$ sucrose in deionized water) and lyophilized. PLGA NP were stored at $-80^{\circ} \mathrm{C}$ until use. Xanthohumol-encapsulating PLGA NP were synthesized by dissolving $5 \mathrm{mg}$ Xanthohumol in the 
initial organic phase. PLGA NP properties were determined by dynamic light scattering using a ZetaSizer analyzer (Malvern Pananalytical Inc., Westborough, MA, USA).

\subsection{Desiccating stress/ scopolamine model for experimental dry eye disease}

All animals were treated in accordance with the ARVO Statement for the Use of Animals in Ophthalmic and Vision Research and the European Commission Directive 86/609/EEC for animal experiments, using protocols approved and monitored by the Animal Experiment Board of Finland. C57BL/6JRj mice were purchased from Janvier Labs (Le Genest-Sainte-Isle, France). Mice were housed at a constant temperature $\left(22 \pm 1^{\circ} \mathrm{C}\right)$ and in a light-controlled environment (lights on from 7 AM to 7 PM) with ad libitum access to food and water. Male mice (9 weeks of age) were used for experiments.

Dry eye disease-like pathology was induced by exposure to a combination of desiccating stress in SiccaSystem ${ }^{\circledR}$ cages (K\&P Scientific LLC, Forest Park, IL, USA) and transdermal administration of scopolamine (Scopoderm ${ }^{\circledR}$; Glaxo Smith Kline, Middlesex, UK), as described by us previously [17, 24]. In this study, mice were exposed to desiccating stress/ scopolamine for a total of 26 days; test articles were administered by twice daily $(8 \mathrm{am}$ and $5 \mathrm{pm})$ topical instillation $(10 \mu \mathrm{l})$ into both eyes starting on day 16 for a period of ten days.

Tear volume was quantified using phenol red-coated threads (ZoneQuick ${ }^{\circledR}$; FCI Ophthalmics, Pembroke, MA, USA), as described by us previously [17, 24].

Corneal fluorescein staining measurements were performed on day 16 and on day 26, as described by us previously [17, 24]. Animals were randomized and assigned to treatment groups based on the corneal fluorescein score on day 16.

\subsection{8-Hydroxy-2'-deoxyguanosine (8-OHdG) staining}

On study day 26 after corneal fluorescein imaging, mice were euthanized by thoracotomy following intraperitoneal administration of $75 \mathrm{mg} / \mathrm{kg}$ ketamine and $1 \mathrm{mg} / \mathrm{kg}$ xylazine. Ocular tissues were fixed in $4 \%$ paraformaldehyde in phosphate buffered saline, cryoprotected in serial sucrose solution $(10 \%, 20 \%, 30 \% \mathrm{w} / \mathrm{v}$ in phosphate-buffered saline) and cryosections of cornea were labeled with an anti-8-OHdG antibody (clone N45.1, 1:200 dilution, Japan Institute for the Control of Aging, NIKKEN SEIL Co., Ltd., Shizuoka, Japan) and analyzed for nuclear staining intensity as described by us in detail previously [17].

\subsection{Data Analysis and Statistics}

All data were analyzed with the investigator blinded for treatment group. Data are presented as mean \pm standard error of mean (SEM) or as median \pm interquartile range or $25^{\text {th }} / 75^{\text {th }}$ percentile. Data were analyzed using paired or unpaired Student's $t$-test, Wilcoxon signed rank test, One-Way analysis of variance (ANOVA) or Kruskal-Wallis ANOVA, or Two-Way ANOVA. Differences between groups were subsequently determined using either Tukey's, Dunn's, or Sidak's multiple comparisons tests as appropriate. Differences were considered statistically significant at the $P<0.05$ level.

\section{Conclusions}

Xanthohumol was cytoprotective against oxidative stress injury in human corneal epithelial cells. Xanthohumol-encapsulating PLGA NP significantly improved dry eye disease pathology in the mouse desiccating stress/ scopolamine model. PLGA NP represent a safe and efficacious drug delivery vehicle for hydrophobic small molecules to the ocular surface. Future studies will optimize Xanthohumol NP-based formulations with the goal to minimize instillation frequency.

Author Contributions: Conceptualization, Anita Ghosh and Simon Kaja; Data curation, Anita Ghosh and Simon Kaja; Formal analysis, Anita Ghosh and Simon Kaja; Funding acquisition, Anita Ghosh, Evan Stubbs Jr, Giedrius Kalesnykas, Jenni Hakkarainen, Kelly Langert and Simon Kaja; Investigation, Anita Ghosh, Rubina Thapa, Harsh Hariani, Michael Volyanyuk, Karoline Orloff, Samatha Ankireddy, Karen Lai, Agne Ziniauskaite, 
Giedrius Kalesnykas, Jenni Hakkarainen, Kelly Langert and Simon Kaja; Methodology, Anita Ghosh, Rubina Thapa, Harsh Hariani, Evan Stubbs Jr, Giedrius Kalesnykas, Jenni Hakkarainen, Kelly Langert and Simon Kaja; Project administration, Anita Ghosh, Giedrius Kalesnykas, Jenni Hakkarainen and Simon Kaja; Resources, Evan Stubbs Jr, Giedrius Kalesnykas and Simon Kaja; Supervision, Giedrius Kalesnykas, Jenni Hakkarainen and Simon Kaja; Validation, Anita Ghosh, Giedrius Kalesnykas, Jenni Hakkarainen and Simon Kaja; Visualization, Anita Ghosh and Simon Kaja; Writing - original draft, Anita Ghosh and Simon Kaja; Writing - review \& editing, Anita Ghosh, Rubina Thapa, Harsh Hariani, Michael Volyanyuk, Karoline Orloff, Samatha Ankireddy, Karen Lai, Agne Ziniauskaite, Evan Stubbs Jr, Giedrius Kalesnykas, Jenni Hakkarainen, Kelly Langert and Simon Kaja All authors have read and agreed to the published version of the manuscript.

Funding: This study was conducted with support from the Illinois Society for the Prevention of Blindness (AKG, HNH), the Dr. John P. and Therese E. Mulcahy Endowed Professorship in Ophthalmology (SK), the Richard A. Perritt M.D. Charitable Foundation (SK) and the United States Department of Veterans Affairs grants \#BX003938 (EBS, SK) and \#RX002305 (KAL). KAO was the recipient of an Association for Pharmacology and Experimental Therapeutics Summer Undergraduate Research Fellowship. This material is the result of work supported with resources and the use of facilities at the Edward Hines Jr. VA Hospital, Hines, IL. The contents do not represent the views of the U.S. Department of Veterans Affairs or the United States Government. Additional support by Experimentica Ltd. and K\&P Scientific LLC is gratefully acknowledged.

Acknowledgments: The authors would like to thank Anne Mari Haapaniemi and Anni Tenhunen for excellent technical support.

Conflicts of Interest: Employment: RT, AŽ, GK, JJH (Experimentica Ltd.); Stock/equity ownership: GK, JJH, SK (Experimentica Ltd.); SK (K\&P Scientific LLC); AKG (eyeNOS Inc.); Consulting: AKG (K\&P Scientific LLC); SK (Experimentica Ltd.). AKG and SK are inventors on a filed patent application on drug targets for dry eye disease, unrelated to this manuscript. SK conducts academic research in areas of interest similar to the business interests of Experimentica Ltd and K\&P Scientific LLC. The terms of this arrangement have been reviewed and approved by Loyola University Chicago in accordance with its conflict of interest policy. The funders had no role in the design of the study; in the collection, analyses, or interpretation of data; in the writing of the manuscript, or in the decision to publish the results.

\section{Abbreviations}

$\begin{array}{ll}\text { 8-OHdG } & \text { 8-hydroxy-2'-deoxyguanosine } \\ \text { ANOVA } & \text { One-Way Analysis of Variance } \\ \text { Keap1 } & \text { Kelch-like ECH-associated protein 1 } \\ \text { LDH } & \text { lactate dehydrogenase } \\ \text { MTT } & \text { 3-(4,5-dimethylthiazol-2-yl)-2,5-diphenyltetrazolium bromide } \\ \text { Mn-TM-2-PyP } & \text { manganese(III) tetrakis(1-methyl-4- pyridyl) porphyrin } \\ \text { NF-kB } & \text { nuclear factor kappa B } \\ \text { NP } & \text { nanoparticle } \\ \text { Nrf2 } & \text { nuclear factor erythroid 2-related factor 2 } \\ \text { PLGA } & \text { poly(lactic-co-glycolic acid) } \\ \text { ROS } & \text { Reactive Oxygen Species } \\ t \text { BHP } & \text { tert-butyl hydroperoxide }\end{array}$

\section{References}

1. Donnenfeld, E.D., et al., Safety of Lifitegrast Ophthalmic Solution 5.0\% in Patients With Dry Eye Disease: A 1-Year, Multicenter, Randomized, Placebo-Controlled Study. Cornea, 2016. 35(6): p. 741-8.

2. Jones, L., et al., TFOS DEWS II Management and Therapy Report. Ocul Surf, 2017. 15(3): p. 575-628.

3. de Paiva, C.S., et al., Topical cyclosporine A therapy for dry eye syndrome. Cochrane Database Syst Rev, 2019. 9: p. CD010051.

4. $\quad$ Seen, S. and L. Tong, Dry eye disease and oxidative stress. Acta Ophthalmol, 2018. 96(4): p. e412-e420.

5. Macri, A., et al., Evaluation of oxidative stress levels in the conjunctival epithelium of patients with or without dry eye, and dry eye patients treated with preservative-free hyaluronic acid $0.15 \%$ and vitamin B12 eye drops. Graefes Arch Clin Exp Ophthalmol, 2015. 253(3): p. 425-30.

6. Choi, W., et al., Expression of Lipid Peroxidation Markers in the Tear Film and Ocular Surface of Patients with Non-Sjogren Syndrome: Potential Biomarkers for Dry Eye Disease. Curr Eye Res, 2016. 41(9): p. 1143-9. 
7. Deng, R., et al., Oxidative stress markers induced by hyperosmolarity in primary human corneal epithelial cells. PLoS One, 2015. 10(5): p. e0126561.

8. Ziniauskaite, A., et al., Manganese(III) tetrakis(1-methyl-4-pyridyl) porphyrin, a superoxide dismutase mimetic, reduces disease severity in in vitro and in vivo models for dry-eye disease. Ocul Surf, 2019.

9. Uchino, Y., et al., A new mouse model of dry eye disease: oxidative stress affects functional decline in the lacrimal gland. Cornea, 2012. 31 Suppl 1: p. S63-7.

10. Uchino, Y., et al., Oxidative stress induced inflammation initiates functional decline of tear production. PLoS One, 2012. 7(10): p. e45805.

11. Wei, Y., et al., The Role of SKQ1 (Visomitin) in Inflammation and Wound Healing of the Ocular Surface. Ophthalmol Ther, 2019. 8(1): p. 63-73.

12. Petrov, A., et al., SkQ1 Ophthalmic Solution for Dry Eye Treatment: Results of a Phase 2 Safety and Efficacy Clinical Study in the Environment and During Challenge in the Controlled Adverse Environment Model. Adv Ther, 2016. 33(1): p. 96-115.

13. Aragona, P., et al., Modern approach to the treatment of dry eye, a complex multifactorial disease: a P.I.C.A.S.S.O. board review. Br J Ophthalmol, 2020.

14. Liu, M., et al., Pharmacological profile of xanthohumol, a prenylated flavonoid from hops (Humulus lupulus). Molecules, 2015. 20(1): p. 754-79.

15. Yao, J., et al., Xanthohumol, a polyphenol chalcone present in hops, activating Nrf2 enzymes to confer protection against oxidative damage in PC12 cells. J Agric Food Chem, 2015. 63(5): p. 1521-31.

16. Langert, K.A., B. Goshu, and E.B. Stubbs, Jr., Attenuation of experimental autoimmune neuritis with locally administered lovastatin-encapsulating poly(lactic-co-glycolic) acid nanoparticles. J Neurochem, 2017. 140(2): p. 334-346.

17. Ziniauskaite, A., et al., Manganese(III) tetrakis(1-methyl-4-pyridyl) porphyrin, a superoxide dismutase mimetic, reduces disease severity in in vitro and in vivo models for dry-eye disease. Ocul Surf, 2019. 17(2): $\mathrm{p}$. 257-264.

18. Baudouin, C., et al., Revisiting the vicious circle of dry eye disease: a focus on the pathophysiology of meibomian gland dysfunction. Br J Ophthalmol, 2016. 100(3): p. 300-6.

19. Lan, W., et al., Nuclear Factor-kappaB: central regulator in ocular surface inflammation and diseases. Ocul Surf, 2012. 10(3): p. 137-48.

20. Lee, H.S., et al., Expression of toll-like receptor 4 contributes to corneal inflammation in experimental dry eye disease. Invest Ophthalmol Vis Sci, 2012. 53(9): p. 5632-40.

21. Becker, U., et al., A comparative evaluation of corneal epithelial cell cultures for assessing ocular permeability. Altern Lab Anim, 2008. 36(1): p. 33-44.

22. Yamasaki, K., et al., Genomic aberrations and cellular heterogeneity in SV40-immortalized human corneal epithelial cells. Invest Ophthalmol Vis Sci, 2009. 50(2): p. 604-13.

23. Dursun, D., et al., A mouse model of keratoconjunctivitis sicca. Invest Ophthalmol Vis Sci, 2002. 43(3): p. 632-8.

24. Ziniauskaite, A., et al., Efficacy of Trabodenoson in a Mouse Keratoconjunctivitis Sicca (KCS) Model for DryEye Syndrome. Invest Ophthalmol Vis Sci, 2018. 59(7): p. 3088-3093.

25. Swetledge, S., et al., Distribution of polymeric nanoparticles in the eye: implications in ocular disease therapy. J Nanobiotechnology, 2021. 19(1): p. 10.

26. Amann, L.C., et al., In vitro-in vivo correlations of scalable PLGA-risperidone implants for the treatment of schizophrenia. Pharm Res, 2010. 27(8): p. 1730-7.

27. Yavuz, B. and U.B. Kompella, Ocular Drug Delivery. Handb Exp Pharmacol, 2017. 242: p. 57-93.

28. Wagh, V.D. and D.U. Apar, Cyclosporine A Loaded PLGA Nanoparticles for Dry Eye Disease: In Vitro Characterization Studies. Journal of Nanotechnology, 2014. 2014: p. 1-10.

29. Ames, P. and A. Galor, Cyclosporine ophthalmic emulsions for the treatment of dry eye: a review of the clinical evidence. Clin Investig (Lond), 2015. 5(3): p. 267-285.

30. Zhang, M., et al., Emerging roles of Nrf2 and phase II antioxidant enzymes in neuroprotection. Prog Neurobiol, 2013. 100: p. 30-47.

31. Ghosh, A.K., et al., Differential Activation of Glioprotective Intracellular Signaling Pathways in Primary Optic Nerve Head Astrocytes after Treatment with Different Classes of Antioxidants. Antioxidants (Basel), 2020. 9(4).

32. Kaja, S., et al., Quantification of Lactate Dehydrogenase for Cell Viability Testing Using Cell Lines and Primary Cultured Astrocytes. Curr Protoc Toxicol, 2017. 72: p. 2 26 1-2 2610.

33. Kaja, S., et al., Plate reader-based cell viability assays for glioprotection using primary rat optic nerve head astrocytes. Exp Eye Res, 2015. 138: p. 159-66. 